\title{
DIRECT-SAFE: A Randomized Controlled Trial of DIRECT Endovascular Clot Retrieval versus Standard Bridging Therapy
}

\author{
Peter J. Mitchell, ${ }^{\mathrm{a}}$ Bernard Yan, ${ }^{\mathrm{b}}$ Leonid Churilov, ${ }^{\mathrm{b}, \mathrm{c}}$ Richard J. Dowling, ${ }^{\mathrm{a}}$ Steven Bush, ${ }^{\mathrm{a}}$ Thang Nguyen, ${ }^{\mathrm{d}}$ \\ Bruce C.V. Campbell, ${ }^{\mathrm{b}, \mathrm{e}}$ Geoffrey A. Donnan, ${ }^{\mathrm{b}}$ Zhongrong Miao, ${ }^{\mathrm{f}, \mathrm{g}}$ Stephen M. Davis, ${ }^{\mathrm{b}}$ on Behalf of the \\ DIRECT-SAFE Investigators \\ a'Department of Radiology, The Royal Melbourne Hospital, University of Melbourne, Parkville, Australia \\ bepartment of Medicine and Neurology, Melbourne Brain Centre at The Royal Melbourne Hospital, University of Melbourne, Parkville, Australia \\ 'Melbourne Medical School, University of Melbourne, Parkville, Australia \\ ${ }^{d}$ Comprehensive Stroke Centre, Department of Neurology, The People's Hospital 115, Pham Ngoc Thach University of Medicine, Ho Chi Minh City, \\ Vietnam \\ 'The Florey Institute of neuroscience and Mental Health, Parkville, Australia \\ fDepartment of Interventional Neuroradiology, Beijing Tiantan Hospital, Capital Medical University, Beijing, China \\ ${ }^{9}$ Department of Neurology, Beijing Tiantan Hospital, Capital Medical University, Beijing, China
}

Background and Purpose The benefit regarding co-treatment with intravenous (IV) thrombolysis before mechanical thrombectomy in acute ischemic stroke with large vessel occlusion remains unclear. To test the hypothesis that clinical outcome of ischemic stroke patients with intracranial internal carotid artery, middle cerebral artery or basilar artery occlusion treated with direct endovascular thrombectomy within 4.5 hours will be non-inferior compared with that of standard bridging IV thrombolysis followed by endovascular thrombectomy.

Methods To randomize 780 patients 1:1 to direct thrombectomy or bridging IV thrombolysis with thrombectomy. An international-multicenter prospective randomized open label blinded endpoint trial (PROBE) (ClincalTrials.gov identifier: NCT03494920).

Results Primary endpoint is functional independence defined as modified Rankin Scale (mRS) $0-2$ or return to baseline at 90 days. Secondary end points include ordinal mRS analysis, good angiographic reperfusion (modified Thrombolysis in Cerebral Infarction score [mTICl] 2b-3), safety endpoints include symptomatic intracerebral hemorrhage and death.

Conclusions DIRECT-SAFE will provide unique information regarding the impact of direct thrombectomy in patients with large vessel occlusion, including patients with basilar artery occlusion, with comparison across different ethnic groups.
Correspondence: Peter J. Mitchell Department of Radiology, The Royal Melbourne Hospital, University of Melbourne, City Campus, Level 1, 300 Grattan Street, Parkville 3050, Australia Tel: +61-3-9342-6450

Fax: +61-3-9342-8369 E-mail: Peter.mitchell@mh.org.au https://orcid.org/0000-0002-8337-7529

Received: October 6, 2021

Revised: December 10, 2021

Accepted: December 27, 2021

Keywords Ischemic stroke; Thrombectomy; Thrombolytic therapy 


\section{Introduction}

\section{Evidence for standard bridging therapy: combined intravenous thrombolysis and endovascular thrombectomy}

The results of five randomized controlled studies comparing intravenous (IV) thrombolysis with combined "bridging" IV thrombolysis and endovascular clot retrieval showed indisputable superiority of the latter. A subsequent pooled analysis (Highly Effective Reperfusion evaluated in Multiple Endovascular Stroke Trials [HERMES]) confirmed the beneficial effects of endovascular clot retrieval which extended across a spectrum of subgroups. ${ }^{1}$ The published guidelines from American Stroke Association and European Stroke Organization recommend endovascular clot retrieval for stroke patients with stroke onset $<6$ hours and large artery occlusion. These guidelines also recommend administration of IV thrombolysis in parallel for those patients who are eligible as this was routine practice in all randomized trials. Endovascular clot retrieval therapy is the current standard of care for ischemic stroke patients with major vessel occlusion, regardless of whether patients are eligible or ineligible for IV thrombolysis.

\section{Intravenous thrombolysis: evidence and potential harm}

The use of IV thrombolysis (with alteplase) is supported by level 1 evidence of improved functional outcome when given within 4.5 hours of stroke onset. ${ }^{2}$ The number needed to treat to achieve excellent functional outcome (modified Rankin Scale [mRS] 0-1) ranges from 4.5 within 90 minutes, to 9 from 90 180 minutes, and 14 from 180-270 minutes. Beyond 4.5 hours, trials in imaging-selected patients have shown benefit up to 24 hours. ${ }^{3}$ Currently, well organized major stroke units treat up to $20 \%$ of acute ischemic stroke patients with standard IV thrombolysis. Although this seems a relatively small percentage, in metropolitan centres the majority of patients with potentially disabling stroke are able to be treated within the 4.5 -hour window. Despite this time limitation and potential contraindications to thrombolysis, the great majority of patients in endovascular trials that allowed enrolment of both thrombolysis eligible and ineligible patients were able to receive standard IV thrombolysis (e.g., 89\% in Multicenter Randomized Clinical Trial of Endovascular Treatment for Acute Ischemic Stroke in the Netherlands [MR CLEAN]). ${ }^{4}$

\section{Potential risks and benefits of intravenous thrombolysis in patients undergoing thrombectomy}

There is evidence in rodent stroke models of ischemia reperfu- sion that alteplase can disrupt the blood-brain barrier (BBB), leading to intracerebral hemorrhage. ${ }^{5}$ The magnitude of BBB disruption appears to be amplified by alteplase and matrix metalloproteinase interactions. ${ }^{6}$ In human studies, the recent Enhanced Control of Hypertension and Thrombolysis Stroke Study (ENCHANTED) trial, comparing low dose with standard dose alteplase, showed an increased proportion of symptomatic intracerebral hemorrhage $(\mathrm{s} I \mathrm{CH})$ in the group treated with standard dose alteplase, suggesting a dose-related toxicity profile for alteplase. ${ }^{7}$ Furthermore, some clinical series examining endovascular treated patients suggested an increased incidence of sICH in those administered IV alteplase, ${ }^{7}$ particularly in those with a large ischemic core. ${ }^{8}$

The risk of $\mathrm{sICH}$ in the endovascular arm of recent randomized trials was 4.4\% overall. ${ }^{1}$ This varied substantially from $0 \%$ in Solitaire ${ }^{\text {TM }}$ With the Intention For Thrombectomy as PRIMary Endovascular Treatment (SWIFT PRIME) ${ }^{9}$ and Extending the Time for Thrombolysis in Emergency Neurological Deficits-Intra-Arterial (EXTEND-IA) ${ }^{10}$ which excluded patients with a large ischemic core, to $6 \%$ in the MR CLEAN trial ${ }^{4}$ which enrolled a relatively unselected group of patients with large vessel occlusion. In current practice, the patients treated as per clinical guidelines more closely resemble MR CLEAN selection criteria. In addition, patients with intracranial atherosclerosis (which is common in Asia and under-represented in the randomized trials) may require endovascular stenting, which requires additional antiplatelet use and may further increase the risk of $\mathrm{sICH}$. The spontaneous rate of $\mathrm{sICH}$ in the placebo arm of IV thrombolysis trials was approximately $1 \%$. There is, therefore, an opportunity to achieve clinically meaningful reduction in the risk of sICH, which is associated with a high rate of death and disability.

It is relatively rare for IV thrombolysis to achieve recanalization before commencement of the endovascular procedure (7.6\% in the HERMES trials ${ }^{1}$ ). Although an effect of IV thrombolysis in facilitating thrombectomy is possible, it is also possible for thrombolysis to cause clot fragmentation and subsequent distal migration that is out of reach of mechanical thrombectomy. The extent of reperfusion has been strongly correlated with clinical outcome and whether the potential removal of distal emboli with thrombolysis outweighs the effect of fragmentation and possible reduction in endovascular success remains uncertain. "In patients receiving IV alteplase, the odds ratio (OR) favoring treatment was 2.45 (95\% confidence intervl $[\mathrm{Cl}], 1.68$ to 3.57$)$ and those treated with "direct" thrombectomy OR was virtually the same, $2.43(95 \% \mathrm{Cl}, 1.3$ to 4.55). ${ }^{1}$ However there were different effects favoring intervention in sub-group analyses. 
Preclinical studies have also suggested a delayed neurotoxic effect exerted by alteplase beyond its thrombolytic properties. Through modulation of several signalling pathways, especially 8 matrix metalloproteinase 9 and $\mathrm{N}$-methyl-D-aspartate receptors, alteplase increased the risk of neurotoxicity and apoptosis. ${ }^{12}$ These effects are difficult to measure in humans but could potentially impact functional outcome.

\section{Methods}

\section{Study design}

DIRECT-SAFE is an international-multicenter prospective randomized open label blinded endpoint trial (PROBE). The treatment arm was direct thrombectomy, the standard care arm was IV thrombolysis with thrombectomy. The trial randomization flow chart is depicted in Figure 1. The trial is an international study conducted in Australia, China, Vietnam, and New Zealand at 25 centers. The first patient was enrolled on June 2, 2018.

\section{Patient population}

The trial population includes patients with vascular imaging confirmed intracranial internal carotid artery (ICA), middle ce-

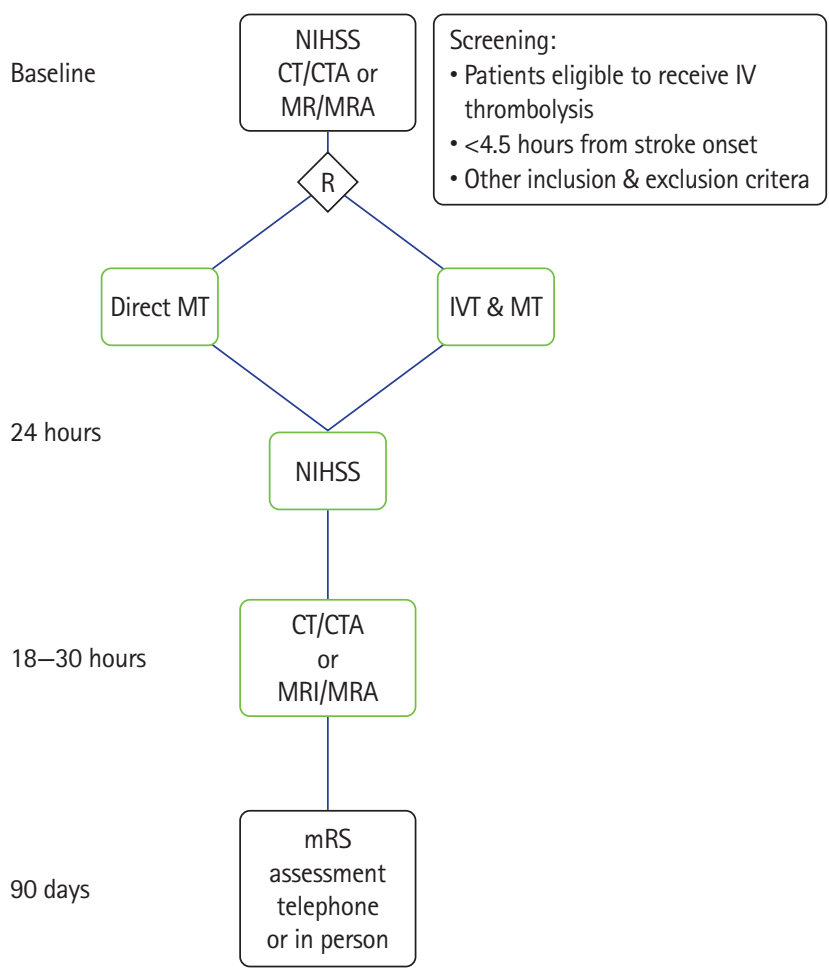

Figure 1. Study assessment flow chart. NIHSS, National Institutes of Health Stroke Scale; CT, computed tomography; CTA, computed tomography angiography; MRA, magnetic resonance angiography; IV, intravenous; $R$, randomization; MT, mechanical thrombectomy; IVT, intravenous thrombolysis; $\mathrm{MRI}$, magnetic resonance imaging; mRS, modified Rankin Scale. rebral artery (MCA) (M1 or $\mathrm{M} 2)$, or basilar artery (BA) large vessel occlusion. Patients must be eligible for IV thrombolysis and stroke onset within 4.5 hours. Endovascular thrombectomy should commence within 90 minutes of randomization. Inclusion and exclusion criteria are included in Table 1.

\section{Randomization and blinding}

Patients should be randomized within 90 minutes of hospital arrival, to either trial arm (treatment or control in ratio 1:1). Randomized patients will be stratified for site of baseline arterial occlusion (ICA, MCA, and BA) and geographic region (Australia/New Zealand vs. Asia).

The allocation of the patient will be disclosed to the interventionist after randomization. An independent and blinded clinician, certified in assessment of the mRS, will undertake assessment of the primary outcome at 90 days. This will be either by clinical visit or telephone review. Imaging data will be evaluated by members of the central imaging core laboratory.

\section{Treatment}

\section{Intravenous thrombolysis}

Patients in the IV thrombolysis (alteplase or tenecteplase) arm will receive IV thrombolysis as per standard care at each site.

Table 1. Trial population

Inclusion criteria
Patients presenting with acute ischemic stroke eligible using standard
criteria to receive IV thrombolysis within 4.5 hours of stroke onset
Age $\geq 18$ years
Intra-arterial clot retrieval treatment can commence (groin puncture)
within 6 hours of stroke onset.
Arterial occlusion on CTA or MRA of the ICA, M1, M2 or basilar artery
Exclusion criteria
Intracranial hemorrhage (ICH) identified by CT or MRI
Rapidly improving symptoms at the discretion of the investigator
Pre-stroke mRS score of $\geq 4$ (indicating previous disability)
Hypodensity in $>1 / 3$ MCA territory on non-contrast CT
Contra indication to imaging with contrast agents
Any terminal illness such that patient would not be expected to survive
more than 1 year
Any condition that, in the judgment of the investigator could impose
hazards to the patient if study therapy is initiated or affect the
participation of the patient in the study.
Pregnant women

IV, intravenous; CTA, computed tomography angiography; MRA, magnetic resonance angiography; ICA, internal carotid artery; CT, computed tomography; $\mathrm{MRI}$, magnetic resonance imaging; $\mathrm{mRS}$, modified Rankin Scale; MCA, middle cerebral artery. 
Vital signs will be recorded during and after the period of infusion as per standard care. The thrombolytic agent used, time of thrombolysis commencement and the dose administered is recorded.

\section{Endovascular therapy}

All patients will then be transferred to the interventional neuroradiology suite with an emphasis on minimizing delays to groin puncture. If dramatic clinical recovery occurs in the interim, the patient should still undergo diagnostic angiography. Recovery does not necessarily imply recanalization and angiography is the best method to establish whether there is an ongoing target for therapy. The use of conscious sedation or general anaesthesia for the procedure is at the investigator's discretion. Close attention should be paid to maintaining stable blood pressure and minimizing delays in starting the procedure. During the procedure, catheters may be flushed with heparinised saline at a concentration of 1,000 units heparin per $1.0 \mathrm{~L}$ $0.9 \%$ sodium chloride. Mechanical thrombectomy as standard of care procedure, will be with the Trevo ${ }^{\circledR}$ device (Stryker Neurovascular, Mountain View, CA, USA) as first line intervention. The decision for proximal balloon guide and aspiration, distal intermediate catheter aspiration or subsequent use of additional catheters or devices is at the discretion of the investigator. Stenting of the extracranial ICA or intracranial atherosclerotic disease is permitted when absolutely necessary to obtain access to distal occlusion or to prevent acute re-occlusion. This may require the use of antiplatelets. Otherwise, no heparin or antiplatelets/anticoagulants should be given until at least 24 hours after the procedure. The initial and final angiograms will be centrally graded by imaging core laboratory for angiographic reperfusion using the modified Thrombolysis in Cerebral Infarction $(\mathrm{mTICl})$ classification and any embolization into new territories. In addition, presence of intracranial atherosclerotic disease will be recorded.

Close neurological observation will be conducted primarily during the first 48 hours after treatment administration according to local clinical practice.

\section{Clinical and imaging evaluation}

Imaging is performed with computed tomography and computed tomography angiography, or magnetic resonance imaging and magnetic resonance angiography acutely as part of standard care with imaging follow-up at 18 to 36 hours. Major vessel occlusion (ICA, MCA, basilar) is required for study eligibility. Where possible, computed tomographic perfusion and magnetic resonance perfusion can be performed at baseline and 18 to 36 hours.
Neurological impairment and functional scores will be measured by a neurologist or health care professional trained in their administration. The assessors will be blinded to the treatment group. The National Institutes of Health Stroke Scale (NIHSS) is a validated neurological impairment score, which will be performed at baseline, then again at 24 hours after treatment (or if initially anaesthetised, as soon as assessable) and day 3. At day 90 ( \pm 7 days), the mRS will be assessed via telephone and adjudicated by a central, blinded panel to assess functional outcome. ${ }^{13}$ An improvement in the NIHSS of $\geq 8$ or final NIHSS $\leq 1$ and an mRS of 0,1 , or 2 are used as indicators of good or excellent outcome in stroke trials.

\section{Primary outcome}

The primary outcome is functional independence defined as mRS $0-2$ or return to baseline at 90 days.

\section{Secondary outcomes}

Secondary outcomes are: (1) mRS 0-1 or return to baseline at 3 months; (2) ordinal shift on mRS at 3 months; (3) proportion of patients with death due to any cause at 3 months; (4) proportion of patients with sICH up to 72 hours post intervention; (5) proportion of patients with good angiographic reperfusion ( $\mathrm{mTICl} 2 \mathrm{~b}-3$ ) at completion of procedure; and (6) proportion of patients with more than 8 points reduction in NIHSS or reaching 0-1 NIHSS at 3 days (early neurological improvement).

\section{Tertiary outcomes}

Tertiary outcomes include: (1) proportion of patients with $\mathrm{sICH}$ in the subgroup with intracranial atherosclerotic disease; (2) $m R S$ ordinal analysis in the subgroup with intracranial atherosclerotic disease; (3) proportion of patients with reperfusion on angiographic examination; (4) proportion of patients with distal embolization post-endovascular clot retrieval; (5) time from hospital admission to $\mathrm{mTICl} 2 \mathrm{~b}-3$ or end of procedure; (6) time from arterial puncture to $\mathrm{mTICl} 2 b-3$ or end of procedure; and (7) median reduction in stroke severity (NIHSS) within 72 hours.

\section{Data Safety Monitoring Board}

An independent Data Safety Monitoring Board (DSMB) includes neurologists and statistician, delegated to convene regularly to monitor progress of the trial.

\section{Sample size calculation}

An estimated total sample size of 780 patients (with 390 patients in each of treatment and control arms) should yield 80\% power to demonstrate non-inferiority in proportions of patients 
achieving primary outcome in the direct group compared with standard bridging therapy, assuming this proportion to be 0.46 and non-inferiority margin of 0.1 at two-sided statistical significance threshold of $P=0.05$.

Adaptive increase in sample size will be performed if the result of interim analysis using data from the first 600 patients is promising as per the methodology of Mehta and Pocock ${ }^{14}$ with a maximum sample size of 900 .

\section{Statistical analysis}

The primary non-inferiority analysis will be based on both an intention-to-treat and per-protocol basis. The protocol specified a non-inferiority margin of -0.1 for the primary outcome of achieving mRS 0-2 or no change from baseline (return to pre-morbid mRS). Non-inferiority would be established if the lower bound of the two-sided 95\% confidence interval (CI) around the difference in proportions of patients who achieved primary outcome between the direct group and the standard bridging therapy group was greater than the pre-defined non-inferiority margin. The two-sided $95 \% \mathrm{Cl}$ around the difference of proportions will be estimated by generating stratum-specific risk differences with corresponding $95 \% \mathrm{Cl}$ for each of the four strata (age $<60$ year-old vs. 60 year-old or above by baseline NIHSS $0-15$ or 16 and above) with subsequent pooling across strata using the Mantel-Haenszel method.

The proportions of mRS 0-2 or no change from baseline and death due to any cause will be compared between the direct group and the standard bridging therapy group of the trial, adjusted for geographical region (Australia/New Zealand vs. Asia), age and baseline NIHSS score using a logistic regression model. The proportions of participants with good angiographic reperfusion ( $\mathrm{mTICl} 2 \mathrm{~b}-3$ ) and sICH will be compared between the two groups adjusted for site of arterial occlusion and geographical region using logistic regression.

The ordinal shift analysis of the mRS secondary outcome will be performed using ordinal logistic regression if the proportional odds assumption is satisfied and otherwise assumption-free ordinal analysis on the full range (0-6) of the mRS. ${ }^{15,16}$ Tertiary outcome analyses will be carried out according to standard statistical principles for comparison of parametric or non-parametric distributions as appropriate. ${ }^{17}$

\section{Interim analysis}

Two safety variables (death or symptomatic hemorrhage within 36 hours of intervention) will be monitored in the safety interim analyses. These analyses will be undertaken when 100 patients have completed the 3-month assessment. It will be con- ducted by an independent DSMB. If there are concerns about the safety of participants, this board will make a recommendation to the trial steering committee about continuing, stopping, or modifying the trial. The Haybittle-Peto procedure for generating early stopping boundaries will be used. To compare the safety of standard bridging therapy versus direct therapy, a composite safety outcome of mortality at 3 months and the incidence of sICH within 36 hours of intervention will be tested. A recommendation of early termination due to external signals, or due to safety reasons will be considered by the independent DSMB if the corresponding Haybittle-Peto boundary $(P=0.001, Z=3)$ at a given interim analysis is crossed.

\section{Study organization funding}

DIRECT-SAFE is an investigator led clinical trial. The sponsor of the trial is the Florey Institute. The trial is supported by a National Health and Medical Research Council (NHMRC) stroke program grant, and Stryker USA (Kalamazoo, MI, USA). The trial is managed by Neuroscience Trials Australia (NTA), Melbourne Australia. Database management and central data monitoring are performed by NTA and independent statistical analysis is performed by the Methods and Implementation Support for Clinical and Health research Hub, The University of Melbourne, Parkville Australia.

\section{Discussion}

DIRECT-SAFE aims to answer whether omission of IV thrombolysis offers similar benefits to patients receiving bridging therapy and thrombectomy. In addition, DIRECT-SAFE is unique in including patients with basilar thrombosis. The benefit of thrombectomy in patients with basilar thrombosis has not been confidently established in randomized trials, and the addition of information regarding outcomes in patients with basilar thrombosis will enrich our knowledge base. Furthermore, DIRECT-SAFE includes patients from disparate ethnic groups (Caucasians, Asians) which increases generalizability and potential influence on clinical practice in our region. Dosing of IV alteplase in the Asian recruiting sites is the standard $0.9 \mathrm{mg} /$ $\mathrm{kg}$, similar to Western population dosing, removing heterogeneity in dosing seen in some treatment centers.

DIRECT-SAFE is one of six randomized studies to test the hypothesis that direct thrombectomy is non-inferior to bridging thrombolysis with thrombectomy. The results of three studies have been published and two others reported in abstract form (Table 2).

The Direct Intra-arterial Thrombectomy in Order to Revascularize AIS Patients With Large Vessel Occlusion Efficiently in 


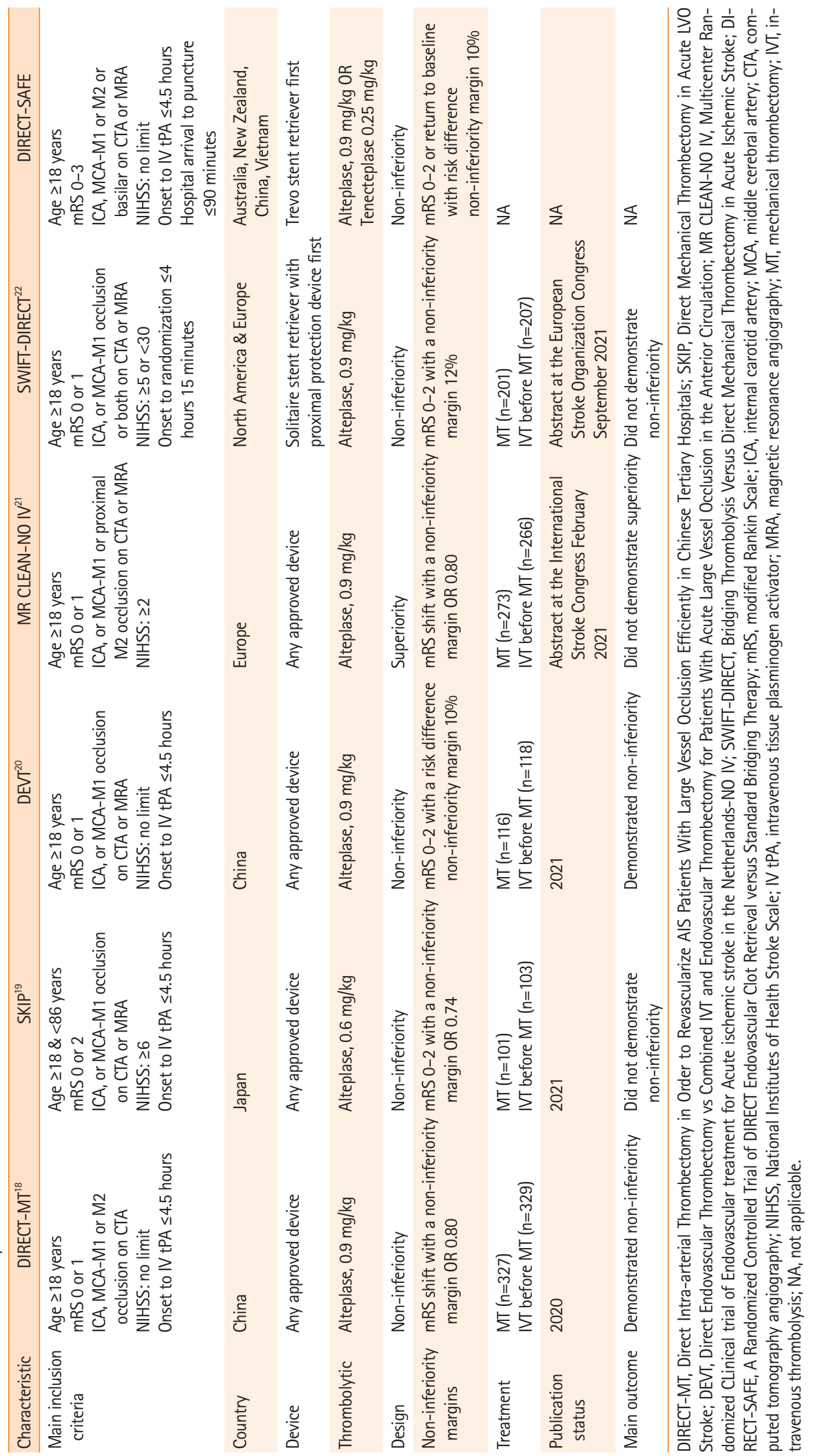


Chinese Tertiary Hospitals (DIRECT-MT) trial recruited 656 acute ischemic stroke patients with large vessel occlusion in the anterior circulation. ${ }^{18}$ The patients recruited were all from China. Direct thrombectomy was judged non-inferior to bridging therapy as the lower bound of the $95 \% \mathrm{Cl}$ did not cross the pre-specified margin of 0.80 (adjusted common odds ratio [cOR], 1.07; 95\% Cl, 0.81 to 1.40). The Direct Mechanical Thrombectomy in Acute LVO Stroke (SKIP) trial recruited 204 patients in Japan. All patients had anterior circulation large vessel occlusion. ${ }^{19}$ Non-inferiority was not demonstrated as the lower boundary of the $95 \% \mathrm{Cl}$ crossed the pre-specified non-inferiority margin of 0.74 (mRS $0-2,59.4 \%$ in the direct thrombectomy group vs. $57 \%$ in the bridging therapy group; $\mathrm{OR}, 1.09$; one-sided $97.5 \% \mathrm{Cl}, 0.63$ to infinity). The Direct Endovascular Thrombectomy vs Combined IVT and Endovascular Thrombectomy for Patients With Acute Large Vessel Occlusion in the Anterior Circulation (DEVT) trial recruited 234 stroke patients from China, also with anterior circulation large vessel occlusion. ${ }^{20}$ This trial was stopped early at interim analysis with $54.3 \%$ in the direct thrombectomy versus $46.6 \%$ in the combined therapy group reaching the primary outcome of $\mathrm{mRS}$ 0-2 at 3 months. The absolute difference was 7.7\% (one sided $97.5 \% \mathrm{Cl}_{1}-5.1 \%$ to infinity), satisfying the non-inferiority margin of $-10 \%$. The Multicenter Randomized CLinical trial of Endovascular treatment for Acute ischemic stroke in the Netherlands-NO IV (MR CLEAN-NO IV) trial recruited 547 stroke patients in three European countries. All patients had anterior circulation large vessel occlusion. Direct thrombectomy was neither superior nor non-inferior (adjusted cOR, 0.88; 95\% Cl, 0.65 to 1.19). ${ }^{21}$ Bridging Thrombolysis Versus Direct Mechanical Thrombectomy in Acute Ischemic Stroke (SWIFT-DIRECT) recruited 408 patients in Europe and did not meet its non-inferiority margin of $12 \%$ (mRS 0-2 57\% direct vs. $65 \%$ bridging, adjusted risk difference $-7.3 \%$; $95 \% \mathrm{Cl}_{1}-17 \%$ to $2.1 \%$ ). ${ }^{22}$

A recent meta-analysis showed that the proportion of patients reaching good clinical outcome (mRS 0-2) was 46\% in DIRECT-MT and $45 \%$ with bridging IV thrombolysis and thrombectomy. The absolute risk difference between the two groups was 1\% favoring direct thrombectomy. In addition the $95 \% \mathrm{Cl}$ of the lower bound was $4 \%$, satisfying a more stringent non-inferiority margin of $5 \%{ }^{23}$

\section{Conclusions}

DIRECT-SAFE will provide valuable additional information in an ethnically diverse population of patients with a broad range of large vessel occlusion sites. Patient level meta-analysis of all six randomized controlled studies may identify subgroups of patients more likely to benefit from a direct or bridging approach to endovascular thrombectomy.

\section{Disclosure}

The trial is supported by Stryker USA. Stryker USA provided an infrastructure grant but had no input into the study design, data collection, data analysis, data interpretation or writing of the protocol and statistical analysis plan.

\section{Acknowledgments}

DIRECT-SAFE is an investigator led clinical trial. The sponsor of the trial is the Florey Institute. The trial is supported by an NHMRC stroke program grant.

\section{References}

1. Goyal M, Menon BK, van Zwam WH, Dippel DW, Mitchell PJ, Demchuk AM, et al. Endovascular thrombectomy after large-vessel ischaemic stroke: a meta-analysis of individual patient data from five randomised trials. Lancet 2016;387: 1723-1731.

2. Emberson J, Lees KR, Lyden P, Blackwell L, Albers G, Bluhmki

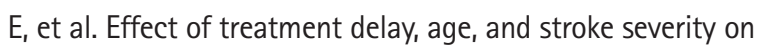
the effects of intravenous thrombolysis with alteplase for acute ischaemic stroke: a meta-analysis of individual patient data from randomised trials. Lancet 2014;384:1929-1935.

3. Ma H, Campbell B, Parsons MW, Churilov L, Levi CR, Hsu C, et al. Thrombolysis guided by perfusion imaging up to 9 hours after onset of stroke. N Engl J Med 2019;380:1795-1803.

4. Berkhemer OA, Fransen PS, Beumer D, van den Berg LA, Lingsma HF, Yoo AJ, et al. A randomized trial of intraarterial treatment for acute ischemic stroke. N Engl J Med 2015;372: $11-20$.

5. Suzuki $Y$, Nagai $N$, Umemura K. A review of the mechanisms of blood-brain barrier permeability by tissue-type plasminogen activator treatment for cerebral ischemia. Front Cell Neurosci 2016;10:2.

6. Wang W, Li M, Chen Q, Wang J. Hemorrhagic transformation after tissue plasminogen activator reperfusion therapy for ischemic stroke: mechanisms, models, and biomarkers. Mol Neurobio/ 2015;52:1572-1579.

7. Anderson CS, Robinson T, Lindley RI, Arima H, Lavados PM, Lee $\mathrm{TH}$, et al. Low-dose versus standard-dose intravenous alteplase in acute ischemic stroke. N Engl J Med 2016;374: 2313-2323.

8. Gilgen MD, Klimek D, Liesirova KT, Meisterernst J, KlingerGratz PP, Schroth G, et al. Younger stroke patients with large 
pretreatment diffusion-weighted imaging lesions may benefit from endovascular treatment. Stroke 2015;46:2510-2516.

9. Saver JL, Goyal M, Bonafe A, Diener HC, Levy El, Pereira VM, et al. Stent-retriever thrombectomy after intravenous t-PA vs. t-PA alone in stroke. N Engl J Med 2015;372:2285-2295.

10. Campbell BC, Mitchell PJ, Kleinig TJ, Dewey HM, Churilov L, Yassi $N$, et al. Endovascular therapy for ischemic stroke with perfusion-imaging selection. N Engl J Med 2015;372:10091018.

11. Coutinho JM, Liebeskind DS, Slater LA, Nogueira RG, Clark W, Dávalos $A$, et al. Combined intravenous thrombolysis and thrombectomy vs thrombectomy alone for acute ischemic stroke: a pooled analysis of the SWIFT and STAR studies. JAMA Neurol 2017;74:268-274.

12. Bhatia R, Hill MD, Shobha N, Menon B, Bal S, Kochar $P$, et al. Low rates of acute recanalization with intravenous recombinant tissue plasminogen activator in ischemic stroke: real-world experience and a call for action. Stroke 2010;41: 2254-2258.

13. McArthur KS, Johnson PC, Quinn TJ, Higgins $P$, Langhorne $P$, Walters MR, et al. Improving the efficiency of stroke trials: feasibility and efficacy of group adjudication of functional end points. Stroke 2013;44:3422-3428.

14. Mehta CR, Pocock SJ. Adaptive increase in sample size when interim results are promising: a practical guide with examples. Stat Med 2011;30:3267-3284.

15. Churilov L, Arnup S, Johns H, Leung T, Roberts S, Campbell $B C$, et al. An improved method for simple, assumption-free ordinal analysis of the modified Rankin Scale using generalized odds ratios. Int J Stroke 2014;9:999-1005.

16. Howard G, Waller JL, Voeks JH, Howard VJ, Jauch EC, Lees $\mathrm{KR}$, et al. A simple, assumption-free, and clinically interpretable approach for analysis of modified Rankin outcomes.
Stroke 2012;43:664-669.

17. Altman DG, Schulz KF, Moher D, Egger M, Davidoff F, Elbourne $D_{\text {, et }}$ al. The revised CONSORT statement for reporting randomized trials: explanation and elaboration. Ann Intern Med 2001;134:663-694.

18. Yang P, Zhang Y, Zhang L, Zhang Y, Treurniet KM, Chen W, et al. Endovascular thrombectomy with or without intravenous alteplase in acute stroke. N Engl J Med 2020;382:1981-1993.

19. Suzuki K, Matsumaru $Y$, Takeuchi M, Morimoto M, Kanazawa $R$, Takayama $Y$, et al. Effect of mechanical thrombectomy without vs with intravenous thrombolysis on functional outcome among patients with acute ischemic stroke: the SKIP randomized clinical trial. JAMA 2021;325:244-253.

20. Zi W, Qiu Z, Li F, Sang H, Wu D, Luo W, et al. Effect of endovascular treatment alone vs intravenous alteplase plus endovascular treatment on functional independence in patients with acute ischemic stroke: the DEVT randomized clinical trial. JAMA 2021;325:234-243.

21. Roos Y. Direct endovascular treatment (dEVT) versus intravenous alteplase followed by endovascular treatment in patients with acute stroke due to a large vessel occlusion. Proceedings of the International Stroke Conference 2021; 2021 Mar 17-19; Online.

22. Fischer U, Gralla J. Solitaire ${ }^{\mathrm{TM}}$ With the Intention For Thrombectomy Plus Intravenous t-PA Versus DIRECT Solitaire ${ }^{\mathrm{TM}}$ Stent-retriever Thrombectomy in Acute Anterior Circulation Stroke (SWIFT DIRECT). Proceedings of the 7th European Stroke Conference; 2021 Sep 1-3; Online.

23. Lin $\mathrm{CH}$, Saver JL, Ovbiagele B, Huang WY, Lee M. Endovascular thrombectomy without versus with intravenous thrombolysis in acute ischemic stroke: a non-inferiority meta-analysis of randomized clinical trials. J Neurointerv Surg 2021 Jul 15 [Epub]. https://doi.org/10.1136/neurintsurg-2021-017667. 